\title{
ASSOCIAÇÃO ENTRE AS VARIÁVEIS SOCIOECONÔMICAS E A AUTOPERCEPÇÃO DE SAÚDE EM IDOSOS COMUNITÁRIOS
}

Janaina Rocha Niehues - Programa de Pós-Graduação em Ciências da Reabilitação - PPGCR da Universidade Federal de Santa Catarina (UFSC) Campus Araranguá, Araranguá - SC, Brasil. Email: jananiehues@gmail.com;

Rafaela Aguiar Rosa - Graduanda do Curso de Fisioterapia na Universidade Federal de Santa Catarina (UFSC) Campus Araranguá, Araranguá - SC, Brasil. Email: rafaelaaguiarrosa14@gmail.com;

Núbia Carelli Pereira de Avelar - Docente do Curso de Fisioterapia do Departamento de Ciências da Saúde do Centro de Ciências, Tecnologias e Saúde do Campus Araranguá. Email: nubia.carelli@ufsc.br;

Ana Lúcia Danielewicz - Programa de Pós-Graduação em Ciências da Reabilitação - PPGCR da

Universidade Federal de Santa Catarina (UFSC) Campus Araranguá, Araranguá - SC, Brasil. Docente do Curso de Fisioterapia do Departamento de Ciências da Saúde do Centro de Ciências, Tecnologias e Saúde do Campus Araranguá. Email: ana.lucia.d@ufsc.br

\section{RESUMO}

Introdução: A autopercepção de saúde (APS) é um importante preditor de morbimortalidade, quedas, institucionalização e sintomas depressivos em idosos. Acredita-se que a baixa renda e menor escolaridade estejam relacionadas com APS negativa, pois esses idosos são mais suscetíveis a condições crônicas e desfechos negativos, sendo importante identificar essa possível associação para auxiliar no planejamento de ações de saúde. Objetivo: Verificar a associação entre variáveis socioeconômicas e a APS em idosos comunitários. Métodos: Tratou-se de um estudo transversal, aleatório, de base domiciliar, com 308 idosos comunitários ( $\geq 60$ anos), do município Balneário Arroio do Silva/SC. A variável desfecho foi a APS avaliada pelo autorrelato e categorizada em autopercepção positiva (muito boa/boa) e negativa (regular/ruim/muito ruim). As variáveis de exposição foram anos de estudo (0-4 anos, 5-9 anos e $\geq 10$ anos) e renda mensal em salários mínimos ( $<1,5$ salários e $\geq 1,5$ salários). Foi realizado o teste de Qui-Quadrado de Pearson. Resultados: A amostra foi composta em sua maioria por mulheres (57,8\%), com média de idade de 69,86 ( $\mathrm{DP}=7,06$ anos). A maior parte dos idosos (56,7\%) relataram ter renda $<1,5$ salários mínimos (IC95\% 5,1;6,2) e 48,3\% tinham de 0-4 anos de estudo (IC95\% 4,2;5,3). A APS foi referida como negativa por 61,3\% dos idosos (IC95\% 5,5;6,6). Na análise bivariada, 117 idosos $(64,2 \%)$ que tinham APS negativa relataram receber renda de até 1,5 salários mínimos $(p<0,001)$. Não foram observadas associações significativas entre os anos de estudo e a APS. Conclusão: Houve associação entre menor renda mensal e APS negativa nos idosos avaliados.

Palavras-chave: renda; escolaridade; autoavaliação. 